\title{
COHERENT HARMONICS IN THE SUPER-RADIANT REGIME FROM AN FEL*
}

\author{
S. V. Benson" ${ }^{\#}$ Joe Gubeli, and M. Shinn, Thomas Jefferson National Accelerator Facility, Newport News, VA, USA
}

\section{Abstract}

Early work that quantified the ratio of coherent harmonic power to the fundamental power as a function of cavity length found that the ratio peaked for a large cavity length detuning. This is consistent with an overbunching model of coherent harmonic production. Recent measurements on the IR Demo FEL at Jefferson Lab have shown that the ratio is also high very near the synchronous point. In this regime the laser is operating a super-radiant mode, similar to what might be present in a SASE device. The recent data is compared to predictions of harmonic production in both long pulse oscillators and SASE FELs.

\section{INTRODUCTION}

Any device that bunches electrons with a small energy spread using a sinusoidal velocity modulation will tend to produce a microscopic density distribution that has Fourier components at all the harmonics of the bunching wavelength. The Fourier component for the fundamental is optimized for slightly stronger bunching than for the harmonics. This effect was seen in microwave electron tubes long before free-electron lasers were developed [1]. Shortly after free-electron lasers began operating in the optical regime, coherent harmonics were detected from them [2]. A detailed study of coherent harmonics was carried out by Deacon and Bamford using the Mark III freeelectron laser at Stanford University [3]. This laser has a short macropulse, which limits operation to at most a few hundred passes. We have recently measured the relative harmonic production in the IR Demo free-electron laser, [4] which is capable of continuous operation. For these measurements it was operated with macropulses allowing 4680 passes through the FEL. As we will show, the behavior near the synchronous detuning differs from what one expects from the overbunching model developed by Deacon and Bamford to explain the Mark III coherent harmonic results.

\section{BACKGROUND}

The analysis and experimental data of Deacon and Bamford was consistent with a model of harmonic generation in which electrons, bunched by the fundamental power, radiate coherently at harmonics of the bunching wavelength. As the fundamental power rises, the harmonic power also rises until a peak is obtained at some fraction of the saturated fundamental power. The

\footnotetext{
* Work supported by the U. S. Department of Energy under contract DE-AC05-84-ER40150, the Office of Naval Research, the Commonwealth of Virginia, and the Laser Processing Consortium \# E-mail felman@jlab.org
}

consequences of this model, verified in the experiment, are the following:

- The rise time of the harmonic $h$ during laser turn-on is $h^{-1}$ times the rise time of the fundamental.

- The peak in the harmonic power occurs at a cavity length detuning away from the synchronous point, where the synchronous point is defined as the cavity length for which the round trip time is equal to the arrival time of the electron pulses.

- The relative spectral width is almost independent of the harmonic number.

- The harmonic power falls off approximately by one order of magnitude per harmonic.

Since the Mark III experiments, a new mode of FEL oscillator operation was demonstrated at FOM in Rinhuizen, the Netherlands [5]. It was shown that the behavior of the FEL quite near the synchronous point exhibited super-radiant scaling behavior. Super-radiance in an FEL oscillator is characterized by the development of optical pulses that are shorter than the electron pulse. As the laser saturates, the pulses develop a characteristic shape that is dependent only on the charge and the cavity losses. The peak power, pulse energy, pulse length and efficiency scale as powers of these two parameters. For example, the efficiency varies as the square root of the ratio of the charge to the cavity losses. The peak power scales as the square of this ratio. This type of behavior was actually first predicted to occur in SASE FELs with long top-hat electron pulses [6]. In fact both cases have the same self-similar pulse structure as the laser saturates.

Recently, with interesting growing in the SASE FEL concept, the idea of coherent harmonic generation extending the short wavelength range of an FEL has garnered much interest. Simulations of a SASE FEL with harmonics were carried out by Freund, Biedron, and Milton [7]. These showed that the harmonic powers radiated in a SASE FEL are much stronger as a fraction of the fundamental power than in the Mark III data. These simulations assumed a single frequency and so do not include any super-radiant effects.

The IR Demo FEL has been described elsewhere [4]. Since it is not limited by heating of copper accelerating structures it is capable of providing a continuous stream of micropulses instead of short macropulses. Even when operated in macropulse mode, allowing access to the laser beam for measurements, the electron beam macropulse length is $250 \mu \mathrm{sec}$. Since the round trip frequency of the optical cavity is $18.7125 \mathrm{MHz}$, there are 4680 round trips of the laser during the macropulse. This is easily long enough to get into the super-radiant regime of operation. The micropulses have been shown to be shorter than the 
electron pulses and so the laser can operate in the superradiant regime.

\subsection{Experimental Layout}

In order to see if super-radiant behavior might have some effect on harmonic behavior, specifically, to see if the ratios predicted in a SASE device might be seen, we measured the macropulse energies for the $3^{\text {rd }}, 5^{\text {th }}, 6^{\text {th }}$, and $7^{\text {th }}$, harmonics while the FEL was operating at 3.15 microns.

The layout of the experimental apparatus is shown in figure 1. The experiments were carried out in one of the User labs in the IR Demo FEL User Facility. Only "alignment mode" beam was used. In alignment mode, the duty cycle of the laser is limited to $0.05 \%$ so that experimenters can work safely around the laser beam. The beam was sent through a calcium fluoride prism to spatially separate the harmonics. The fundamental and harmonics were allowed to propagate approximately 4 meters from one optical bench to another to allow them to be cleanly separated. The fundamental was measured using a high energy pyroelectric detector (Molectron model J25). The harmonics were measured using an uncalibrated low energy pyroelectric detector (Eltec model 420-0). The detectors were cross-calibrated by measuring the harmonic signals on both detectors. The response for these harmonics was still measurable on the high energy detector with some amplification. This allowed us to have a good relative measure of the harmonic energy per macropulse. An iris was used to block out any but the harmonic being measured. Calibrated filters were used to further block any stray fundamental light from getting into the harmonic detector.

For each harmonic, the energy per macropulse at was measured a s a function of cavity length. The ratio of the harmonic energy to the fundamental energy was then calculated.

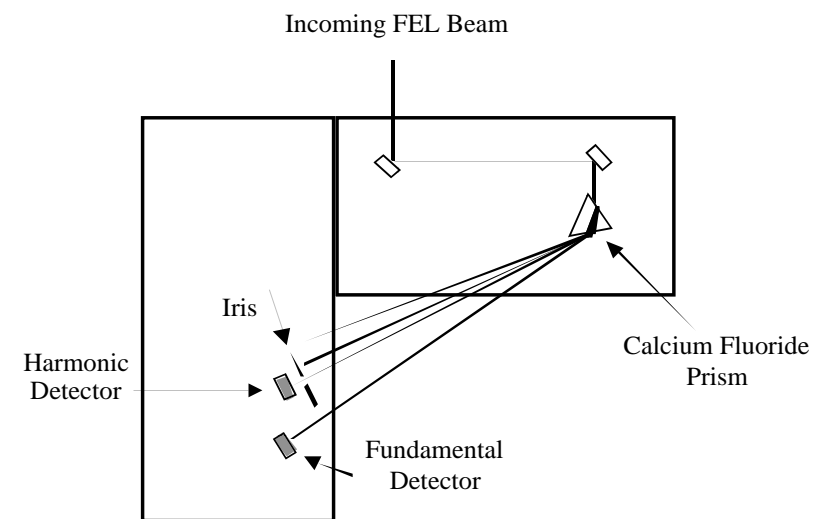

Figure 1. Experimental layout for measuring the macropulse energy of each harmonic relative to the fundamental macropulse energy.

\section{EXPERIMENTAL RESULTS}

In figure 2 we show the macropulse energy in the harmonics and the fundamental as a function of cavity length. The harmonic energy in each harmonic has been normalized to the peak energy vs. cavity length for each harmonic. The synchronous point (cavity length detuning equal to zero) was set to the peak in the fundamental power curve

In figure 3 we show the ratio of the harmonic energy to the fundamental energy as a function of cavity length. Note the logarithmic scale. The signal-to-noise ratio was quite good for the low power detector even for the $6^{\text {th }}$ harmonic. The signal-to-noise ratio for the third harmonic was quite good for the high energy detector. The linearity of both detectors is known to be excellent.

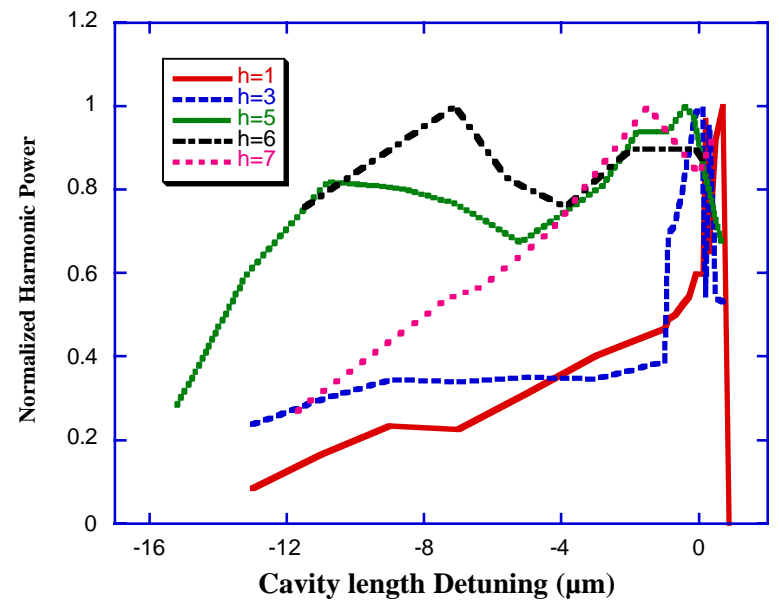

Figure 2. Harmonic macropulse energy relative to the peak energy for that harmonic as a function of cavity length.

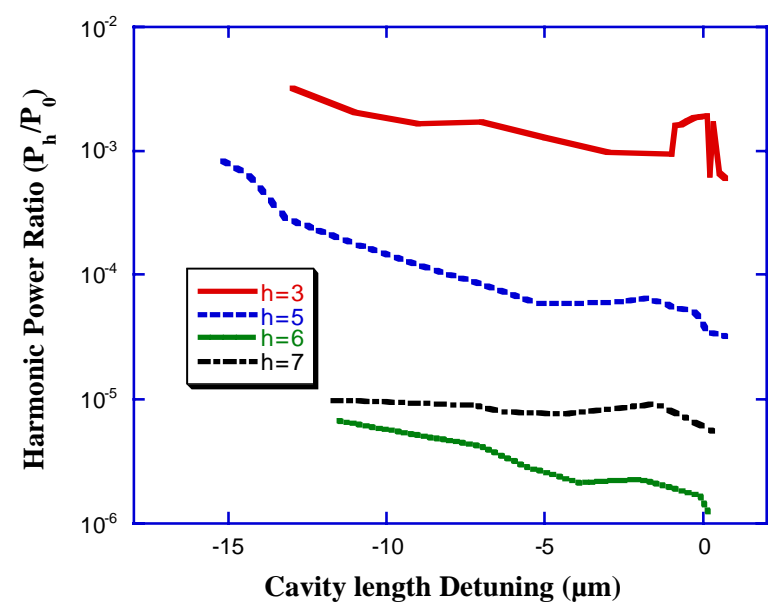

Figure 3. Ratio of the macropulse energy at each harmonic to that of the fundamental as a function of cavity length detuning.

The measured harmonic energies were corrected for the losses in the optical transport system calculated from the known losses vs. wavelength of the silver coated transport mirrors. The transport losses at the third and fifth harmonics were measured and found to agree with the calculated values to better than 5\%. The loss at the $7^{\text {th }}$ harmonic is more uncertain due to the strong dependence 
of the total loss on the mirror reflectivity. It is probably good to $\pm 20 \%$.

\subsection{Discussion}

The fundamental detuning curve in figure 2 has a typical shape for long pulse operation. The curve is approximately triangular with a sharp peak near the synchronous point. From Deacon and Bamford's work we would expect that the power would peak further out on the detuning curve for each higher harmonic and that the ratio should be very low near the synchronous point. In fact, the curves for the $5^{\text {th }}$ and $6^{\text {th }}$ harmonics do have peaks at large detuning. But they then have a second peak near the synchronous point. The other harmonics actually increase monotonically with increasing cavity length. The ratio for most of the harmonics has the expected behavior at large detuning of a decreasing ratio. At small detuning the curves either flatten out or, in the case of the third harmonic, have a large increase. This is exactly opposite to what one might expect from the overbunching model. One possible explanation is that the self-similar bunches present in super-radiant operation have a more effective coherent harmonic production mechanism that that of a long pulse.

The original purpose of this paper was to study the harmonic power ratios in the super-radiant regime and compare to SASE predictions. In fact the ratios are higher than those of the Mark III study and are as much as a factor of ten higher than the theoretical predictions in reference [3]. Since the harmonic production rate is sensitive to the energy spread, the difference might be due solely to the different energy spreads in the two machines. The ratios are quite a bit smaller than those predicted for a SASE FEL. In fact the ratios near the synchronous point are approximately the geometric mean of the Mark III theoretical predictions and the SASE predictions for the ANL device described in reference [7]. Again, the SASE device has yet smaller energy spread, which may be the source of the higher harmonics, especially the very high harmonics. When super-radiant behavior is included in a SASE device however the ratio of coherent harmonic radiation to fundamental radiation may be even larger than in reference [7].

\section{CONCLUSIONS}

We have shown evidence that harmonics in a freeelectron laser oscillator are enhanced over what one might expect from a simple overbunching model appropriate to long pulse operation. The harmonic ratios, despite being much stronger than those reported in previous oscillators, are still close to an order of magnitude less than those predicted for SASE devices. The coherent harmonic enhancement may be due to the nature of the self-similar pulses in the FEL during super-radiant operation. It would be useful to characterize this behavior more fully since SASE devices will probably also show super-radiant behavior.

\section{ACKNOWLEDGEMENTS}

The IR Demo laser operations are the work of many dedicated, hard-working personnel at Jefferson Lab. Work supported by the U. S. Department of Energy under contract DE-AC05-84-ER40150, the Office of Naval Research, the Commonwealth of Virginia, and the Laser Processing Consortium

\section{REFERENCES}

[1] See e.g. the Radiation Lab Series on Klystrons and Traveling Wave Tube amplifiers.

[2] "Review of Recent Experimental Results from the Stanford $3 \mu \mathrm{m}$ Free-Electron Laser", S. Benson et al., Proceedings of the Bendor Free Electron Laser Conference, J. de Physics Coll. (1982) Cl-353.

[3] "Harmonic Generation Experiments on the Mark III Free-Electron Laser", D. J. Bamford and D. A. G. Deacon, Nucl. Inst. and Meth. A296 (1990) 89.

[4] "Sustained Kilowatt Lasing in a Free-Electron Laser with Same-Cell Energy Recovery", G. R. Neil et al., Phys. Rev. Lett., 84, (2000) 662.

[5] "Observation of Superradiance in a Short-Pulse FEL Ocsillator", D. A. Jaroszynski et al., Nucl. Inst. and Meth. A393 (1997) 332.

[6] "The Superradiant Regime of a FEL: Analytical and Numerical Results", R. Bonifacio, L. DeSalvo, P. Pierini, and N. Piovella, Nucl. Inst. and Meth. A 296 (1990) 358.

[7] "Nonlinear Harmonic Generation and Proposed Experimental Verification in SASE FELs" H. P. Freund, S. G. Biedron, and S. V. Milton, Nucl. Inst. and Meth. $\mathbf{4 4 4 5}$ (2000) 53. 